Tohoku J. Exp. Med., 2008, 214, 7-10

\title{
Short Bowel Syndrome: Amelioration of Diarrhea after Vagotomy and Pyloroplasty for Peptic Hemorrhage
}

\author{
Michael Safioleas, ${ }^{1}$ Michael Stamatakos, ${ }^{1}$ Panagiotis Safioleas, ${ }^{1}$ \\ Ahmad Diab, ${ }^{1}$ Evridiki Karanikola ${ }^{1}$ and Constantinos Safioleas ${ }^{1}$ \\ ${ }^{1}$ Second Department of Propaedeutic Surgery, Medical School, University of Athens, Laiko \\ General Hospital, Athens, Greece
}

Acute mesenteric ischemia is a rare symptomatic manifestation of arteriosclerosis. Prognosis crucially depends on rapid diagnosis and surgical management to prevent or at least minimize the bowel infarction. The length of the small bowel is considered to be between 3 and $8 \mathrm{~m}$, and a normal bowel function can be maintained even after resection of its one third. But loss of a major part $(>60 \%)$ can lead to malnutrition and death. However, patients, who survived an extended intestinal resection due to improved postoperative care (intensive care unit and parenteral nutrition), develop short bowel syndrome. This phenomenon is a medical problem, and several surgical techniques have been used to slow down intestinal transit time or to increase the area of absorption. All these procedures have controversial outcomes and are still on different experimental levels; namely, they cannot be recommended for routine use. In our report of a patient suffering from short bowel syndrome, vagotomy and pyloroplasty were performed to repair a sudden peptic hemorrhage. This operation cured bleeding peptic ulcer and also palliated the diarrhea, a main clinical manifestation of short bowel syndrome. In this study, our aim is to emphasize the favorable clinical outcome of vagotomy concerning a principal manifestation of short bowel syndrome, such as diarrhea. To the best of our knowledge, the present study is the first report showing the vagotomy as a possible procedure for the treatment of diarrhea, although this occurrence has no clear explanation. We also discuss the management of short bowel syndrome. - short bowel syndrome; vagotomy; bowel infarction; mesenteric ischemia.

Tohoku J. Exp. Med., 2008, 214 (1), 7-10. (C) 2008 Tohoku University Medical Press

Acute mesenteric ischemia is a rare symptomatic manifestation of arteriosclerosis. Prognosis crucially depends on rapid diagnosis and surgical management to prevent or at least minimize the bowel infarction, which is caused by mesenteric vascular diseases of both the occlusive and nonocclusive types. Delay in diagnosis and treatment of a potentially ischemic intestine is associated with increased mortality that varies in rate from 60 to $100 \%$ (with increased mortality, ranging from 60-100\%) (Kasirajan et al. 2004; Safioleas et al. 2006). Therefore, acute mesenteric ischemia usually results in extensive enterectomy. Patients surviving extended intestinal resection (up to $60-70 \%$ of the small bowel) develop short bowel syndrome (SBS) (Thompson et al. 2005), which is defined as the malabsorptive state following massive small bowel resection

Received August 30, 2007; revision accepted for publication November 17, 2007.

Correspondence: Prof. Michael C. Safioleas, M.D., Ph.D., 7 Kyprou Ave, 15237, Filothei, Athens, Greece.

e-mail: stamatakosmih@yahoo.gr 
(Vanderhoof and Langnas 1997). Thus this syndrome occurs when the length of the residual bowel is smaller than $2 \mathrm{~m}$ (Ruchman et al. 2003). This syndrome is clinically characterized by diarrhea with impaired absorption of fats, vitamins and other nutrients, ultimately leading to malnutrition, anemia and continued weight loss. In some instances, severe gastric hypersecretion also occurs occasionally with the development of duodenal ulcer and its complications (Mitchell et al. 1984; Wang et al. 1999). Finally, SBS is a serious medical problem, as a significant number of patients survive due to improvement of perioperative care (surgeon's ability, intensive care unit, and parenteral nutrition), and several surgical techniques have been used to slow down intestinal transit time or to increase the area of absorption (Herrington and Sawers 1968; Wales 2004). Techniques for the deceleration of intestinal transit, such as reconstruction of the ileocecal valve, antiperistaltic intestinal segments, colon interposition, recirculating intestinal loops and intestinal pacing have been described (Craft and Venables 1968). Growth of neomucosa, mucosal autotransplantation, intestinal lengthening and small bowel transplantation have been performed in an attempt to increase the area of absorption (Goulet and Ruemmele 2006). All these procedures either have controversial outcomes or are still on different experimental levels, and therefore, they cannot be recommended for routine use.

In this report, we describe a patient with SBS, who was successfully treated by vagotomy. Unexpectedly, vagotomy can palliate the diarrhea, a main clinical manifestation of SBS.

\section{Clinical Findings of the Patient}

A 65-year-old male, with no medical history, was operated on, in emergency, due to acute mesenteric ischemia. After massive enterectomy extending from $60 \mathrm{~cm}$ distal to the ligament of Treitz to the mid-transverse colon, an end-to-end jejuno-transverse colostomy was performed. The postoperative course was uneventful and the patient was placed on parenteral nutrition with 2,400 calories daily. Fluids and electrolytes were replaced, balanced with output plus maintenance.
Stools gradually increased to eight on the 6th postoperative day, while on the 12th postoperative day bowel movements were ten with 2,300 $\mathrm{ml}$ in stools. Moreover steatorrhea was present (fat in feces). Even though it seems early, parenteral nutrition was discontinued on the 16th postoperative day and the patient was alimented completely orally with dry meals believing that dietary adjustment could benefit the patient, as parenteral nutrition has a potential role on diarrhea. The patient was given loperamide to relieve his diarrhea. The patient was discharged from the hospital on the 24th postoperative day weighting $68 \mathrm{~kg}$, a 7-kg loss over the preoperative weight. Four days after his discharge, the patient was readmitted to the hospital presenting severe peptic hemorrhage with bloody stools and hematemesis, while he was under treatment with $\mathrm{H} 2$ receptor antagonists (in hindsight a medical approach by proton-pump inhibitors might have been more

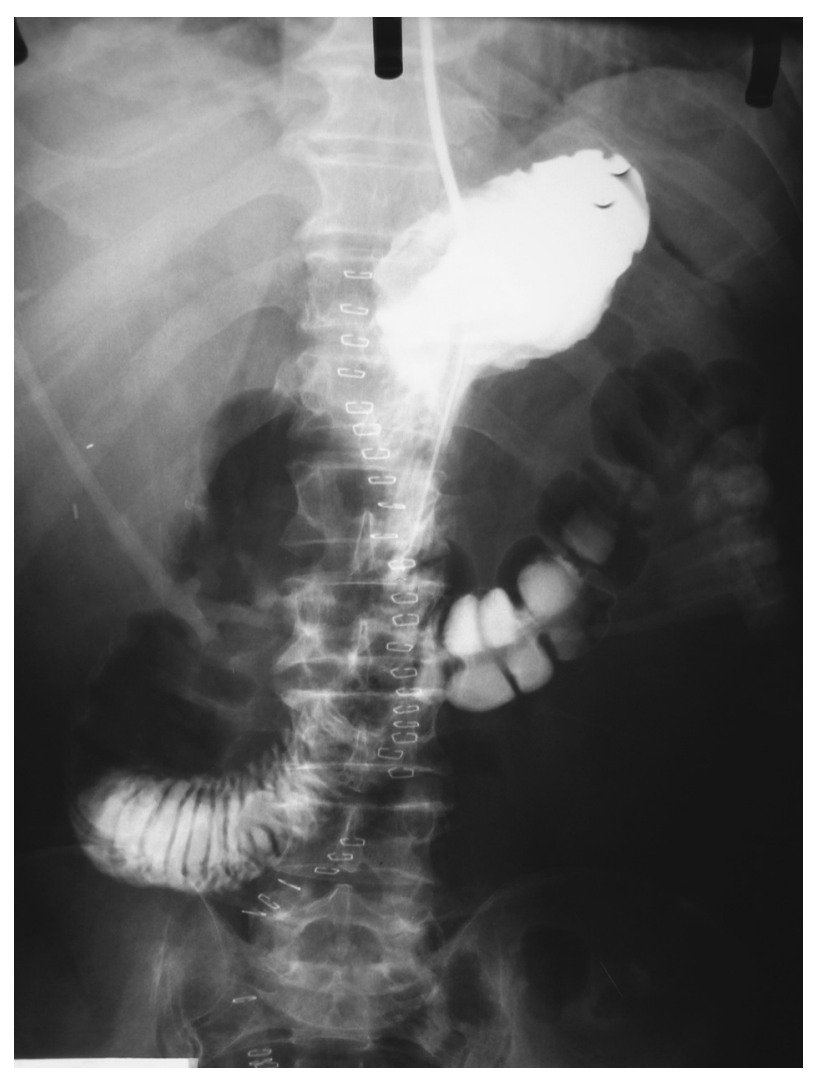

Fig. 1. Plain abdominal radiograph after barium meal. Note the drainage of barium almost directly into the large bowel. 
beneficial). Urgent endoscopy revealed a duodenal ulcer. The patient was treated conservatively but five days later he presented recurrence of hemorrhage and surgical therapy was deemed mandatory. At operation apart from ligation of the bleeding vessel, pyloroplasty associated with truncal vagotomy was performed as a curative approach to duodenal ulcer. The postoperative course was without any serious complications, except for a low grade fever for 3 days. The surprising event was the decreased number of stools to 5-6 per day. Fourteen months after the second operation the patient had gained body weight (1 $\mathrm{kg}$ ), reported increased appetite and the imaging tests showed a good functioning gastrointestinal tract (Fig. 1).

\section{Discussion}

Acute superior mesenteric vascular occlusion resulting in mesenteric ischemia is the most common cause of massive intestinal resection. The improvement of surgical skill, postoperative medical treatment (parenteral nutrition) and hospital care (intensive care unit) have led to decreased mortality rates of massive enterectomy; thus, the necessity of an effective treatment for patients with SBS has become imperative and the management of patients with SBS has to be undertaken by a multidisciplinary team including gastroenterology, surgery and nutrition among other specialties. SBS is more likely to follow ileac resection, since the jejunum is capable of less functional adaptation. Disability is greater if the ileocecal valve is lost and further aggravated by loss of a part of the colon (Kvietys 1999). The essential defects giving rise to SBS following massive enterectomy are the severe shortage of absorptive mucosa and the marked reduction in intestinal length. As a consequence, transit time is rapid and mucosal contact time greatly reduced, with resultant massive fluid and electrolyte losses and insufficient absorption of nutrients. Clinical manifestations of SBS are frequent diarrhea, malnutrition and significant weight loss. The management of such patients requires maintenance of fluid electrolytes and nitrogen balance that can sufficiently be achieved by total or supplementary intravenous nutrition.

After the 1960s the introduction of parenteral nutrition has helped these patients to survive for the first period, giving time to the intestine to adapt and improve the absorptive capacity of the remaining part (adaptation phase). Thus, a significant number of patients with small intestinal remnants become progressively independent of parenteral nutrition and require diet rich in energy supply (Allard and Jeejeebhoy 1989), which should be distributed over multiple small meals including proper supplementation of vitamins and trace elements (maintenance phase) (Keller et al. 2004). However, steatorrhea and diarrhea remain principal manifestations of SBS. Several surgical techniques have been used to slow down intestinal transit time or to increase the area of absorption. Surgical procedures to slow intestinal transit including reconstruction of the ileocecal valve, antiperistaltic intestinal segments, colon interposition, recirculating intestinal loops and intestinal pacing or intestinal valves have been described in case reports or small-case series only. The type of procedure will defined by age, length and functionality of the remnant bowel, the existence of intestinal dilation and the presence of bacterial overgrowth. For the increase of the area of absorption growth of neomucosa, mucosal autotransplantation, intestinal lengthening and small bowel transplantation have been performed (Grant 1999; Goulet and Ruemmele 2006). Concerning the surgical techniques, apart from intestinal loop lengthening advised by Bianchi (1980), which had been successfully used on an infant by Boeckman and Traylor (1981) and was modified by Aigrain et al. (1985), intestinal transplantation appears to be the only alternative for patients who have developed significant liver disease (no cirrhosis) related to short bowel syndrome (Fishbein et al. 2003). Certainly, all these procedures have controversial outcomes and are still at different experimental levels. Therefore, they cannot be recommended for routine use.

The favorable result of vagotomy and pyloroplasty, which we performed in order to face a sudden peptic hemorrhage in the patient with SBS, has no clear explanation except for delay in 
gastric emptying and decrease of acid hypersecretion which increases bowel motility. It has been well established in adult subjects, both clinically and in the laboratory, that marked gastric hypersecretion occurs following major loss of small intestine (Frenderick et al. 1965; Reul and Ellison 1966). Moreover, this outcome supports an experimental study by Randolph in enterectomized puppies also subjected to vagotomy and pyloroplasty, which had appeared a conspicuous decrease of diarrhea one week after vagotomy (Randolph and Lilly 1968). It has been demonstrated that complete vagotomy results in diarrhea (Dragsted et al. 1947). Thus, when we were going to perform vagotomy, we had in mind the possible increase of diarrhea, which in our patient with SBS was already existed.

Moreover, it is well known that after vagotomy and pyloroplasty the duodenal regulation of gastric emptying is lost (McKelvey 1970). The stomach empties under the influence of posture, exposing the intestine to the rapid influx of food, mainly if the meal is fluid. In conclusion, the favorable effect of vagotomy on frequency of diarrhea in SBS, although at first sight paradox, might be of interest. This fact, to our knowledge, has no clear pathophysiologic mechanism and the subject remains open for research and discussion among all physicians concerned.

\section{References}

Aigrain, Y., Cornet, D., Cezard, J.P. \& Boureau, M. (1985) Longitudinal division of small intestine: a surgical possibility for children with the very short bowel syndrome. $L$. Kinderchir, 40, 233-236.

Allard, J.P. \& Jeejeebhoy, K.N. (1989) Nutritional support and therapy in the short bowel syndrome. Gastroenterol. Clin. North Am., 18, 589-601.

Bianchi, A. (1980) Intestinal loop lengthening- a technique for increasing small intestinal length. J. Pediatr. Surg., 15, 145-151.

Boeckman, C.R. \& Traylor, R. (1981) Bowel lengthening for short bowel syndrome. J. Pediatr. Surg., 16, 996-997.

Craft, I.L. \& Venables, C.W. (1968) Antiperistaltic segment of jejunum for persistent diarrhea following vagotomy. Ann. Surg., 167, 282-286.

Dragsted, L.R., Harrer, P.V., Tovee, B.E. \& Woodward, E.R. (1947) Section of the vagus nerves to the Stomach in the treatment of peptic ulcer. Ann. Surg., 126, 687-708.

Fishbein, T.M., Kaufman, S.S. \& Florman, S.S. (2003) Isolated intestinal transplantation: proof of clinical efficacy. Transplantation, 76, 636-640.

Frederick, P.L., Sizer, J.S. \& Osborne, M.P. (1965) Relation of invasive bowel resection to gastric secretion. New Engl. J. Med., 272, 509-514.

Goulet, O. \& Ruemmele, F. (2006) Causes and management of intestinal failure in children. Gastroenterology, 130 (2 Suppl.), 516-528.

Grant, D. (1999) Intestinal transplantation: 1997 report of the international registry. Transplantation, 67, 1061-1064.

Herrington, J.L. \& Sawers, J.L. (1968) A new operation for the dumping syndrome and post-vagotomy diarrhea. Ann. Surg., 168, 522-541.

Kasirajan, K., Mascha, E.J., Heffernan, D. \& Sifuentes, J. (2004) Determinants of in-hospital mortality and length of stay for acute intestinal gangrene. Am. J. Surg., 187, 482-485.

Keller, J., Panter, H. \& Layer, P. (2004) Management of the short bowel syndrome after extensive small bowel resection. Pract. Res. Gastroenterol., 18, 977-992.

Kvietys, P.R. (1999) Intestinal physiology relevant to short bowel syndrome. Eur. J. Pediatric. Surg., 9, 196-199.

McKelvey, S.T.D. (1970) Gastric incontinence and postvagotomy diarrhea. Br. J. Surg., 57, 741-747.

Mitchell, A., Watkins, R.M. \& Collin, J. (1984) Surgical treatment of the short bowel syndrome. Br. J. Surg., 71, 329-333.

Randolph, J.G. \& Lilly, J.R. (1968) The influence of vagotomy and pyloroplasty on the growth and survival of enterectomized young animals. J. Pediatr. Surg., 3, 232-237.

Reul, G.J. \& Ellison, E.H. (1966) Effect of 75\% distal small bowel resection on gastric acid secretion. Am. J. Surg., 111, 772-776.

Ruchman, A.L., Scolapio, J. \& Fryer, J. (2003) AGA technical review on short bowel syndrome and intestinal transplantation. Gastroenterology, 124, 1111-1134.

Safioleas, M.C., Moulakakis, K.G., Papavassiliou, V.G., Kontzoglou, K. \& Kostakis, A. (2006) Acute mesenteric ischaemia, a high lethal disease with devasting outcome. Vasa, 35, 106-111.

Thompson, J.S., DiBase, J.K., Yeats, M. \& Sudan, D.L. (2005) Postoperative short bowel syndrome. J. Am. Coll. Surg., 201, 85-89.

Wales, P. (2004) Surgical therapy for short bowel syndrome. Pediat. Surg. Int., 20, 647-657.

Vanderhoof, J.A. \& Langnas, A.N. (1997) Short bowel syndrome in children and adults. Gastroenterology, 113, $1767-1778$.

Wang, H.T., Miller, J.H., Avissar, N. \& Sax, H.C. (1999) Small bowel adaptation in dependent on site massive enterectomy. J. Surg. Res., 84, 94-100. 